\title{
A Compact CRB for the Single Source Conditional Signal Model with Application to Delay-Doppler-Phase Estimation of Band-Limited Signals
}

\author{
L. Ortega, D. Medina, J. Vilà-Valls, F. Vincent and E. Chaumette
}

\begin{abstract}
The derivation of tight estimation lower bounds is a key player to design and assess the performance of new estimators. In this contribution, we derive a new compact CramérRao bound (CRB) for the conditional signal model, where the deterministic parameter's vector includes a real positive amplitude and the signal phase. Then, such CRB is particularized to the delay, Doppler, phase and amplitude estimation with bandlimited (narrowband) signals, where transmitter and receiver are in relative uniform radial movement. The latter expression is especially easy to use because it only depends on the signal samples. We provide illustrative results for a representative Global Navigation Satellite System positioning example.
\end{abstract}

\section{INTRODUCTION}

In many practical problems of interest dealing with deterministic parameters estimation, such as radar, sonar or navigation, the observations are complex circular vectors [1]. An important estimation problem is the identification of the components of a noisy observation vector $\mathbf{x}$ formed from a linear superposition of $Q$ sources [2]-[5]

$$
\mathbf{x}=\mathbf{A}(\boldsymbol{\eta}) \boldsymbol{\alpha}+\mathbf{n}, \mathbf{x}, \mathbf{n} \in \mathbb{C}^{N}, \mathbf{A}(\boldsymbol{\eta}) \in \mathbb{C}^{N \times Q}, \boldsymbol{\alpha} \in \mathbb{C}^{Q},
$$

where the mixing matrix depends on an unknown deterministic parameter vector $\boldsymbol{\eta} \in \mathbb{R}^{P}$. This problem has received considerable attention during the last fifty years, both for time-series analysis [4] and array processing [5], and merged into the framework of modern array processing [1], [5] where typically two different signal models are considered: the conditional signal model (CSM) and the unconditional signal model [3]. In this article we adopt the less constrained CSM framework. Indeed, assuming that sources are temporally white Gaussian processes is a strong hypothesis that may fail in many real-life applications, i.e., Global Navigation Satellite Systems (GNSS) which ignited this contribution.

Despite nearly optimal properties (in the asymptotic regime, i.e., in the large sample regime [3] and/or high signal-to-noise (SNR) regime [6]) of conditional maximum likelihood estimators (CMLEs), these estimators suffer from a large computational cost, as they require solving a nonlinear multidimensional optimization problem. To circumvent this problem, several suboptimal techniques have been introduced: i) substituting the multidimensional search for

This research was partially supported by the DGA/AID projects (2019.65.0068.00.470.75.01, 2018.60.0072.00.470.75.01) and the TéSA Lab Postdoctoral Research Fellowship.

L. Ortega is with Telecommunications for Space and Aeronautics Lab (TéSA), Toulouse, France; D. Medina is with Institute of Communications and Navigation, German Aerospace Center (DLR), Neustrelitz, Germany; J. Vilà-Valls, F. Vincent and E. Chaumette are with ISAE-SUPAERO, University of Toulouse, Toulouse, France. a simpler one-dimension search, e.g., Capon or MUSIC methods [7], ii) restricting to a single source search, e.g., CLEAN [8] or Alternating Projection algorithms [9], or iii) exploiting the EXtended Invariance Principle (EXIP) [10], which is based on a re-parametrization of the problem that simplifies the ML criterion to be maximized. In EXIP, the efficiency property of the original ML is maintained (at least asymptotically) through a Weighted Least Square (WLS) refining step by using a matched weighting matrix.

EXIP has been used in array [11] and/or radar [12] processing, and lately in GNSS [13]. For instance in GNSS, the EXIP is applied to the ML direct position estimation (DPE) [14] and leads to the widespread suboptimal two-step approach: i) first, estimate delays and Dopplers from each satellite independently, then ii) estimate the user position and velocity thanks to a WLS minimization. If a precise positioning is required (i.e., in intelligent transportation systems or safety-critical applications), the solution is to exploit, together with delay and Doppler, the signal phase information as well [15], [16]. It is thus of the outmost importance to characterize the first step (i.e., delay, Doppler and phase estimation) CMLE asymptotic performance associated to the following single source CSM,

$$
\mathbf{x}=\mathbf{a}(\boldsymbol{\eta}) \alpha+\mathbf{n}, \mathbf{x}, \mathbf{n} \in \mathbb{C}^{N}, \mathbf{a}(\boldsymbol{\eta}) \in \mathbb{C}^{N}, \alpha \in \mathbb{C} .
$$

The CMLE's asymptotic performance in the mean square error (MSE) sense is accurately described by the CramérRao bound (CRB). So, it is not surprising that several CRB expressions for the single source estimation problem have been derived, for finite [17]-[21] or infinite [22] bandwidth signals, where the starting point is often either the SlepianBangs fomulas [23] or general theoretical CRB expressions for Gaussian observation models [5], [7], [24]. However, in the case where in addition to $\boldsymbol{\eta}$ we must estimate amplitude and phase, that is, when $(2 a)$ is reparameterized as

$$
\mathbf{x}=\mathbf{a}(\boldsymbol{\eta}) \rho e^{j \varphi}+\mathbf{n}, \mathbf{x}, \mathbf{n} \in \mathbb{C}^{N}, \mathbf{a}(\boldsymbol{\eta}) \in \mathbb{C}^{N}, \rho \in \mathbb{R}^{+},
$$

to the best of our knowledge, no compact CRB formula for the joint estimation of $\boldsymbol{\epsilon}^{T}=\left(\sigma_{n}^{2}, \rho, \varphi, \boldsymbol{\eta}^{T}\right)$, where $\sigma_{n}^{2}$ is the white noise $\mathbf{n}$ power $\left(\mathbf{n} \sim \mathcal{C} \mathcal{N}\left(\mathbf{0}, \sigma_{n}^{2} \mathbf{I}_{N}\right)\right)$, seems to be available in the open literature [3]-[5], [7], [17]-[32]. This lack is all the more surprising that the derivation of such compact $\mathrm{CRB}$ is not difficult with the ad hoc approach, as proposed in Sec. II, being the first contribution of the article. A noteworthy feature of this new compact CRB is its easeof-use for problems where the CRBs on $\boldsymbol{\eta}$ and $\alpha$ (complex amplitude instead of amplitude and phase) have already been 
computed. A more demanding problem is the extension of preliminary results recently derived in [33] for the timedelay estimation assuming a constant transmitter to receiver propagation delay (i.e., no Doppler effect, static scenario) to the joint delay, Doppler, phase and amplitude estimation case, being the second contribution of this article in Sec. III. Indeed the latter is a fundamental task in a plethora of applications, therefore a tractable CRB for this problem is a key tool of broad interest. In this contribution, we introduce a new compact CRB expression for this problem considering the standard narrowband signal model where the Doppler effect on the band-limited baseband signal is not considered and amounts to a frequency shift. The new compact CRB obtained is expressed in terms of the signal samples, making it especially easy to use whatever the baseband signal considered. Illustrative results are given for a representative GNSS positioning example.

\section{A Compact CRB For the Single Source CSM}

First, we provide some new results on the CRB for the CSM. If we reparameterize (2b) as

$$
\mathbf{x}=\mathbf{a}^{\prime}(\boldsymbol{\theta}) \rho+\mathbf{n}, \mathbf{a}^{\prime}(\boldsymbol{\theta})=\mathbf{a}(\boldsymbol{\eta}) e^{j \varphi}, \boldsymbol{\theta}^{T}=\left(\varphi, \boldsymbol{\eta}^{T}\right),
$$

then the corresponding CRB for $\epsilon$ is given by ${ }^{1}$

$$
\begin{aligned}
& \mathbf{C R B}_{\boldsymbol{\theta}}=\left[\begin{array}{cc}
C R B_{\varphi} & \mathbf{C R B}_{\boldsymbol{\eta}, \varphi}^{T} \\
\mathbf{C R B}_{\boldsymbol{\eta}, \varphi} & \mathbf{C R B}_{\boldsymbol{\eta}}
\end{array}\right] \\
& \mathbf{C R B}_{\boldsymbol{\eta}}=\frac{\sigma_{n}^{2}}{2 \rho^{2}} \operatorname{Re}\left\{\left(\frac{\partial \mathbf{a}(\boldsymbol{\eta})}{\partial \boldsymbol{\eta}^{T}}\right)^{H} \mathbf{\Pi}_{\mathbf{a}(\boldsymbol{\eta})}^{\perp} \frac{\partial \mathbf{a}(\boldsymbol{\eta})}{\partial \boldsymbol{\eta}^{T}}\right\}^{-1}, \\
& C R B_{\varphi}=\frac{\sigma_{n}^{2}}{2 \rho^{2}} \frac{1}{\|\mathbf{a}(\boldsymbol{\eta})\|^{2}} \\
& +\frac{\operatorname{Im}\left\{\mathbf{a}(\boldsymbol{\eta})^{H} \frac{\partial \mathbf{a}(\boldsymbol{\eta})}{\partial \boldsymbol{\eta}^{T}}\right\} \mathbf{C R B}_{\boldsymbol{\eta}} \operatorname{Im}\left\{\mathbf{a}(\boldsymbol{\eta})^{H} \frac{\partial \mathbf{a}(\boldsymbol{\eta})}{\partial \boldsymbol{\eta}^{T}}\right\}^{T}}{\|\mathbf{a}(\boldsymbol{\eta})\|^{4}} \\
& \mathbf{C R B}_{\boldsymbol{\eta}, \varphi}=-\mathbf{C R B}_{\boldsymbol{\eta}} \frac{\left.\operatorname{Im}\{\boldsymbol{\eta})^{H} \frac{\partial \mathbf{a}(\boldsymbol{\eta})}{\partial \boldsymbol{\eta}^{T}}\right\}^{T}}{\|\mathbf{a}(\boldsymbol{\eta})\|^{2}} \\
& C R B_{\rho}=\frac{\sigma_{n}^{2}}{2\|\mathbf{a}(\boldsymbol{\eta})\|^{2}} \\
& +\rho^{2} \frac{\operatorname{Re}\left\{\mathbf{a}(\boldsymbol{\eta})^{H} \frac{\partial \mathbf{a}(\boldsymbol{\eta})}{\partial \boldsymbol{\eta}^{T}}\right\}^{T} \mathbf{C R B} \boldsymbol{\eta} \operatorname{Re}\left\{\mathbf{a}(\boldsymbol{\eta})^{H} \frac{\partial \mathbf{a}(\boldsymbol{\eta})}{\partial \boldsymbol{\eta}^{T}}\right\}}{\|\mathbf{a}(\boldsymbol{\eta})\|^{4}} \\
& C R B_{\sigma_{n}^{2}}=\frac{1}{N}\left(\sigma_{n}^{2}\right)^{2} .
\end{aligned}
$$

Proof: see Appendix I.

Surprisingly, to the best of our knowledge, the compact CRB formulas (3a)-(3f) for the joint estimation of $\epsilon^{T}=$ $\left(\sigma_{n}^{2}, \rho, \varphi, \boldsymbol{\eta}^{T}\right)$ do not seem to have been released in the open literature. A noteworthy feature of this compact CRB is its ease-of-use for problems where the CRBs on $\boldsymbol{\eta}$ and

${ }^{1}$ Let $S=\operatorname{span}(\mathbf{A})$, with $\mathbf{A}$ a matrix, be the linear span of the set of its column vectors, $S^{\perp}$ the orthogonal complement of the subspace $S, \Pi_{\mathbf{A}}=$ $\mathbf{A}\left(\mathbf{A}^{H} \mathbf{A}\right) \mathbf{A}^{H}$ the orthogonal projection over $S$, and $\boldsymbol{\Pi}_{\mathbf{A}}^{\perp}=\mathbf{I}-\boldsymbol{\Pi}_{\mathbf{A}}$. $\alpha$ (the complex amplitude instead of amplitude and phase) have already been computed. Indeed, since $\mathbf{a}(\boldsymbol{\eta})^{H} \frac{\partial \mathbf{a}(\boldsymbol{\eta})}{\partial \boldsymbol{\eta}^{T}}$ naturally appears to compute $\left(\frac{\partial \mathbf{a}(\boldsymbol{\eta})}{\partial \boldsymbol{\eta}^{T}}\right)^{H} \boldsymbol{\Pi}_{\mathbf{a}(\boldsymbol{\eta})}^{\perp} \frac{\partial \mathbf{a}(\boldsymbol{\eta})}{\partial \boldsymbol{\eta}^{T}}$ in (3b), the $\mathrm{CRB}$ for these problems can be readily updated in order to incorporate (3c)-(3e). As an example, we consider the well-known single tone $(\eta)$ estimation problem for which $\mathbf{a}(\eta)=\left(1, e^{j 2 \pi \eta} \ldots, e^{j 2 \pi \eta(N-1)}\right)^{T}[25]:\|\mathbf{a}(\eta)\|^{2}=N$ and

$$
\begin{aligned}
& \mathbf{a}(\eta)^{H} \frac{\partial \mathbf{a}(\eta)}{\partial \eta}=j 2 \pi P, \frac{\partial \mathbf{a}(\eta)^{H}}{\partial \eta} \frac{\partial \mathbf{a}(\eta)}{\partial \eta}=(2 \pi)^{2} Q \\
& P=\sum_{n=0}^{N-1} n=\frac{N(N-1)}{2} \\
& Q=\sum_{n=0}^{N-1} n^{2}=\frac{N(N-1)(2 N-1)}{6}
\end{aligned}
$$

which yields

$$
\left\{\begin{array}{l}
C R B_{\eta}=\frac{\sigma_{n}^{2}}{2 \rho^{2}} \frac{1}{(2 \pi)^{2}\left(Q-\frac{P^{2}}{N}\right)}=\frac{\sigma_{n}^{2}}{\rho^{2}} \frac{6}{N\left(N^{2}-1\right)} \frac{1}{(2 \pi)^{2}} \\
C R B_{\varphi}=\frac{\sigma_{n}^{2}}{2 \rho^{2}} \frac{1}{N-\frac{P^{2}}{Q}}=\frac{\sigma_{n}^{2}}{\rho^{2}} \frac{2 N-1}{N(N+1)} \\
C R B_{\rho}=\frac{\sigma_{n}^{2}}{2 N}
\end{array}\right.
$$

\section{StANDARD NARROWBAND Signal MOdEL}

Given a generic band-limited signal $c(t)$ with bandwidth $B$, it can be expressed in time and frequency as

$$
\begin{aligned}
& c(t)=\sum_{n=N_{1}}^{N_{2}} c\left(n T_{s}\right) \operatorname{sinc}\left(\pi F_{s}\left(t-n T_{s}\right)\right) \rightleftharpoons \\
& c(f)=\left(T_{s} \sum_{n=N_{1}}^{N_{2}} c\left(n T_{s}\right) e^{-j 2 \pi f n T_{s}}\right) 1_{\left[-\frac{B}{2}, \frac{B}{2}\right]}(f),
\end{aligned}
$$

where $F_{s} \geq B, c\left(n T_{s}\right)$ are the samples of $c(t), N_{1}, N_{2} \in \mathbb{Z}$, $N_{1} \leq N_{2}$ and $\rightleftharpoons$ refers to the time-frequency pair. We consider the transmission of this band-limited signal $c(t)$ over a carrier frequency $f_{c}\left(\lambda_{c}=c / f_{c}\right)$, from a transmitter $\mathrm{T}$ to a receiver $\mathrm{R}$. Both transmitter and receiver are in uniform linear motion, that is, their respective positions evolve as $\mathbf{p}_{T}(t)=\mathbf{p}_{T}+\mathbf{v}_{T} t$ and $\mathbf{p}_{R}(t)=\mathbf{p}_{R}+\mathbf{v}_{R} t$ In that perspective, we tackle the simplified case where the propagation delay $\tau(t)$ due to the relative radial movement between $\mathrm{T}$ and $\mathrm{R}$, can be approximated by a first order distance-velocity model,

$$
\begin{aligned}
& \left\|\mathbf{p}_{T R}(t)\right\| \triangleq\left\|\mathbf{p}_{R}(t)-\mathbf{p}_{T}(t-\tau(t))\right\|=c \tau(t) \simeq d+v t \\
& \Rightarrow \tau(t) \simeq \tau+b t, \quad \tau=\frac{d}{c}, \quad b=\frac{v}{c} .
\end{aligned}
$$

This so-called relative uniform radial movement is characterized by the time-delay $(\tau)$ due to the propagation path and the dilation $(1-b)$ induced by the Doppler effect. Under the narrowband hypothesis, i.e. $B \ll f_{c}$, the Doppler effect on the band-limited baseband signal $c(t)$ may be considered negligible. In this case, for an ideal transmitter, propagation channel and receiver, the signal at the output of the receiver's Hilbert filter (I/Q demodulation, bandwidth $\left.F_{s}\right)$ is well approximated as $[17,(2.1)][21,(3)]$ :

$$
\begin{aligned}
& x(t) \triangleq x(t ; \boldsymbol{\eta})=\alpha c(t-\tau) e^{-j \omega_{c} b(t-\tau)}+n(t), \\
& R_{n}(\tau)=\sigma_{n}^{2} \operatorname{sinc}\left(\pi F_{s} \tau\right) \rightleftharpoons R_{n}(f)=\frac{\sigma_{n}^{2}}{F_{s}}, f \in\left[-\frac{F_{s}}{2}, \frac{F_{s}}{2}\right]
\end{aligned}
$$


where $\omega_{c}=2 \pi f_{c}, \boldsymbol{\eta}^{T}=(\tau, b)$, and $\alpha$ is a complex amplitude which includes all the transmission budget terms. If we consider the acquisition of $N^{\prime}=N_{2}^{\prime}-N_{1}^{\prime}+1$ $\left(N_{1}^{\prime} \ll N_{1}, N_{2}^{\prime} \gg N_{2}\right)$ samples at $T_{s}=1 / F_{s}$, then the discrete vector signal model is given by (2a), or equivalently (2b), with

$$
\begin{aligned}
& \mathbf{x}^{T}=\left(\ldots, x\left(n^{\prime} T_{s}\right), \ldots\right), \\
& \mathbf{a}(\boldsymbol{\eta})^{T}=\left(\ldots, c\left(n^{\prime} T_{s}-\tau\right) e^{-j \omega_{c} b\left(n^{\prime} T_{s}-\tau\right)}, \ldots\right), \\
& \mathbf{n}^{T}=\left(\ldots, n\left(n^{\prime} T_{s}\right), \ldots\right), \\
& \mathbf{c}^{T} \triangleq\left(\ldots, c\left(n T_{s}\right), \ldots\right),
\end{aligned}
$$

for $N_{1}^{\prime} \leq n^{\prime} \leq N_{2}^{\prime}$ and $N_{1} \leq n \leq N_{2}$. The $\mathrm{SNR}$ at the output of the CMLE is defined as

$$
S N R_{\text {out }}=\frac{\alpha^{2} \mathbb{E}}{\left(\frac{\sigma_{n}^{2}}{F_{s}}\right)}=\frac{\alpha^{2} \mathbf{c}^{H} \mathbf{c}}{\sigma_{n}^{2}}, \quad \mathbb{E}=\frac{\mathbf{c}^{H} \mathbf{c}}{F_{s}},
$$

and the CRB for the estimation of $\boldsymbol{\epsilon}^{T}=\left(\sigma_{n}^{2}, \rho, \varphi, \boldsymbol{\eta}^{T}\right)$ is

$$
\begin{aligned}
& \mathbf{C R B}_{\boldsymbol{\eta}}=\frac{1}{2 S N R_{\text {out }}} \boldsymbol{\Delta}_{\boldsymbol{\eta}}^{-1}, \\
& {\left[\boldsymbol{\Delta}_{\boldsymbol{\eta}}\right]_{1,1}=F_{s}^{2}\left(\frac{\mathbf{c}^{H} \mathbf{V} \mathbf{c}}{\mathbf{c}^{H} \mathbf{c}}-\left|\frac{\mathbf{c}^{H} \boldsymbol{\Lambda} \mathbf{c}}{\mathbf{c}^{H} \mathbf{c}}\right|^{2}\right)} \\
& {\left[\boldsymbol{\Delta}_{\boldsymbol{\eta}}\right]_{2,2}=\frac{\omega_{c}^{2}}{F_{s}^{2}}\left(\frac{\mathbf{c}^{H} \mathbf{D}^{2} \mathbf{c}}{\mathbf{c}^{H} \mathbf{c}}-\left(\frac{\mathbf{c}^{H} \mathbf{D} \mathbf{c}}{\mathbf{c}^{H} \mathbf{c}}\right)^{2}\right)} \\
& {\left[\boldsymbol{\Delta}_{\boldsymbol{\eta}}\right]_{1,2}=\left[\boldsymbol{\Delta}_{\boldsymbol{\eta}}\right]_{2,1}=\omega_{c} \operatorname{Im}\left\{\frac{\mathbf{c}^{H} \mathbf{D} \boldsymbol{\Lambda} \mathbf{c}}{\mathbf{c}^{H} \mathbf{c}}-\frac{\mathbf{c}^{H} \mathbf{D} \mathbf{c}}{\mathbf{c}^{H} \mathbf{c}} \frac{\mathbf{c}^{H} \boldsymbol{\Lambda} \mathbf{c}}{\mathbf{c}^{H} \mathbf{c}}\right\}}
\end{aligned}
$$

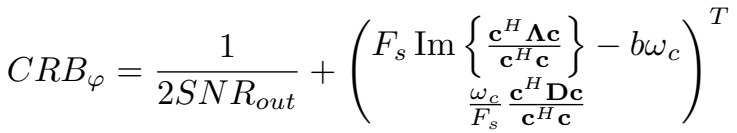

$$
\begin{aligned}
& \times \mathbf{C R B}_{\boldsymbol{\eta}}\left(\begin{array}{c}
F_{s} \operatorname{Im}\left\{\begin{array}{c}
\left.\frac{\mathbf{c}^{H} \boldsymbol{\Lambda} \mathbf{c}}{\mathbf{c}^{H} \mathbf{c}}\right\} \\
\frac{\omega_{c}}{F_{s}} \frac{\mathbf{c}^{H} \mathbf{D c}}{\mathbf{c}^{H} \mathbf{c}}
\end{array}\right), b \omega_{c}
\end{array}\right) \\
& \mathbf{C R B}_{\boldsymbol{\eta}, \varphi}=\mathbf{C R B}_{\boldsymbol{\eta}}\left(\begin{array}{c}
F_{s} \operatorname{Im}\left\{\frac{\mathbf{c}^{H} \boldsymbol{\Lambda} \mathbf{c}}{\mathbf{c}^{H} \mathbf{c}}\right\}-b \omega_{c} \\
\frac{\omega_{c}}{F_{s}} \mathbf{c}^{H} \mathbf{D} \mathbf{c} \\
\mathbf{c}^{H} \mathbf{c}
\end{array}\right), \\
& C R B_{\rho}=\frac{1}{2 \frac{\mathbb{E} F_{s}}{\sigma_{n}^{2}}} \\
& +\rho^{2} F_{s}^{2}\left(\begin{array}{c}
\operatorname{Re}\left\{\frac{\mathbf{c}^{H} \boldsymbol{\Lambda} \mathbf{c}}{\mathbf{c}^{H} \mathbf{c}}\right\} \\
0
\end{array}\right)^{T} \mathbf{C R B}_{\boldsymbol{\eta}}\left(\begin{array}{c}
\operatorname{Re}\left\{\frac{\mathbf{c}^{H} \boldsymbol{\Lambda} \mathbf{c}}{\mathbf{c}^{H} \mathbf{c}}\right\} \\
0
\end{array}\right), \\
& C R B_{\sigma_{n}^{2}}=\frac{1}{N}\left(\sigma_{n}^{2}\right)^{2} \text {, }
\end{aligned}
$$

with $\mathbf{D}, \boldsymbol{\Lambda}$ and $\mathbf{V}$ defined as

$$
\begin{aligned}
\mathbf{D} & =\operatorname{diag}\left(\left[N_{1}, N_{1}+1, \ldots, N_{2}-1, N_{2}\right]\right), \\
(\mathbf{V})_{n, n^{\prime}} & =\mid \begin{array}{l}
n^{\prime} \neq n:(-1)^{\left|n-n^{\prime}\right|} \frac{2}{\left(n-n^{\prime}\right)^{2}} \\
n^{\prime}=n: \frac{\pi^{2}}{3}
\end{array} \\
(\boldsymbol{\Lambda})_{n, n^{\prime}} & =\mid \begin{array}{l}
n^{\prime} \neq n: \frac{(-1)^{\left|n-n^{\prime}\right|}}{\left(n-n^{\prime}\right)} \\
n^{\prime}=n: 0
\end{array}
\end{aligned}
$$

Proof: see Appendix II.

\section{ILlustrative Results: GNSS \\ Delay/Phase-BASED Positioning}

Standard GNSS positioning relies on the use of ML delay/Doppler estimates. In contrast, precise navigation (i.e., such as Real-Time Kinematics (RTK) or Precise Point Positioning (PPP) techniques) [15] relies on the exploitation of the signal phase information. Indeed, the phase is linked to the wavelength which is much smaller than the baseband signal resolution. In GNSS, the use of phase measurements is typically decoupled from the baseband signal processing, i.e., delay/Doppler/phase estimation, and the covariance of these estimates is set somehow empirically, i.e., based on the satellite elevation or the estimated carrier-to-noise density $\left(C / N_{0}\right)$ at the receiver. Therefore, so far it has not been possible to correctly characterize the performance and ultimate achievable precision of carrier phase-based positioning.

Using the new delay/phase compact CRB in Sec. III, which only depends on the signal samples, we are able to assess this delay/phase precision using different GNSS signals. We consider: i) GPS L1 C/A signal, which uses a BPSK modulation at $1.023 \mathrm{MHz}$, ii) GPS L5 signal with a BPSK modulation 10 times faster, and iii) Galileo E5 signal which uses a large bandwidth AltBOC modulation. The delay/phase MLEs are denoted $\widehat{\tau}$ and $\widehat{\varphi}(\widehat{\tau})$, respectively. The phase CRB in [m] is obtained as $\frac{\lambda}{2 \pi} \sqrt{\mathrm{CRB}_{\varphi \mid \underline{\epsilon}}}$, and we use as a reference $\lambda_{E 5}=25.15 \mathrm{~cm}$.

The delay estimation CRB and MLE results for the three signals are shown in Fig. 1. It is clear that using fast codes (i.e., larger bandwidth and narrower autocorrelation function w.r.t. GPS L1 C/A) may significantly improve the delay estimation. This is particularly true for the Galileo E5 signal, for which the gain in delay standard deviation is a factor 28 and 8 w.r.t. GPS L1 and L5, respectively.

The CMLEs values obtained for different $\mathrm{SNR}_{\text {out }}$, considering a $C / N_{0}=45 \mathrm{~dB}-\mathrm{Hz}$, are summarized in Table I. It is remarkable that the phase estimation $\mathrm{CRB}_{\varphi \mid \underline{\epsilon}}$ is $\simeq \frac{1}{2 \mathrm{SNR}_{\text {out }}}$, which implies that it does not depend on the broadcasted signal, as opposite to the delay estimation, and therefore using fast codes do not improve the phase estimation w.r.t. the legacy GPS L1 C/A signal. The huge advantage of using new GNSS signals in terms of delay estimation precision (i.e., see the difference between $\widehat{\tau}$ L1 C/A and $\widehat{\tau}$ E5 in Table I) does not translate to the corresponding signal phase. This supports the fact that AltBOC-type signals can be a promising solution for code-based precise positioning, because the advantage brought by joint delay/phase positioning using these signals may not be worth the inherent complexity and fundamental limitations of phase ambiguity resolution techniques [15], which are the bottleneck of carrier phase-based solutions such as PPP and RTK. These results open the door to new precise positioning receiver design.

\section{CONClusion}

In this article we derived a new compact CRB for the conditional signal model, in order to properly take into account the signal phase and a positive real amplitude. These results were particularized to the case of delay, Doppler, 


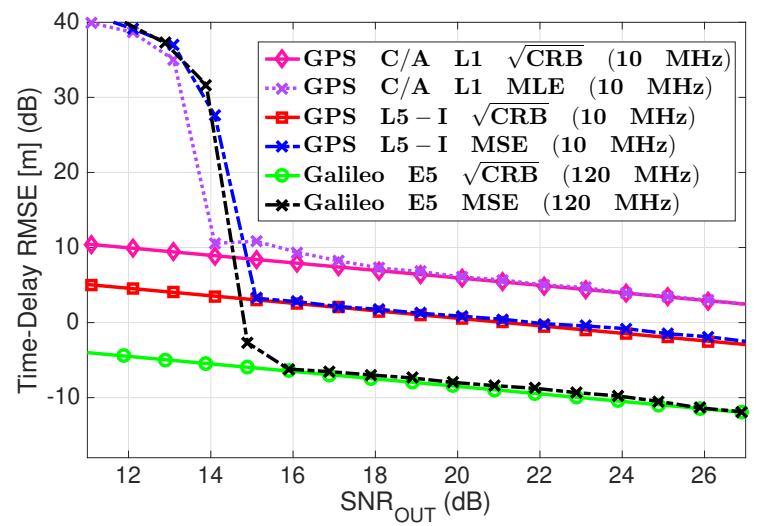

Fig. 1: Time-delay CRB/MLE for GPS L1 C/A BPSK(1), GPS L5-I BPSK(10) and Galileo E5 AltBOC(15,10).

\begin{tabular}{|c|c|c|c|c|c|}
\hline SNR $_{\text {out }}$ & $T_{I}$ & $\widehat{\varphi}(\widehat{\tau})$ & $\widehat{\tau}$ L1 C/A & $\widehat{\tau}$ L5 & $\widehat{\tau}$ E5 \\
\hline 15 & $1 \mathrm{~ms}$ & $5 \mathrm{~mm}$ & $7.08 \mathrm{~m}$ & $2.03 \mathrm{~m}$ & $25 \mathrm{~cm}$ \\
\hline 18 & $2 \mathrm{~ms}$ & $3.6 \mathrm{~mm}$ & $5.01 \mathrm{~m}$ & $1.44 \mathrm{~m}$ & $18 \mathrm{~cm}$ \\
\hline 21 & $4 \mathrm{~ms}$ & $2.5 \mathrm{~mm}$ & $3.55 \mathrm{~m}$ & $1.02 \mathrm{~m}$ & $13 \mathrm{~cm}$ \\
\hline 25 & $10 \mathrm{~ms}$ & $1.6 \mathrm{~mm}$ & $2.24 \mathrm{~m}$ & $64 \mathrm{~cm}$ & $8 \mathrm{~cm}$ \\
\hline 28 & $20 \mathrm{~ms}$ & $1.1 \mathrm{~mm}$ & $1.59 \mathrm{~m}$ & $46 \mathrm{~cm}$ & $6 \mathrm{~cm}$ \\
\hline
\end{tabular}

TABLE I: Phase and Time-delay Estimation Standard Deviation for: GPS L1 C/A $\left(F_{s}=10 \mathrm{MHz}\right), \mathrm{L} 5\left(F_{s}=10\right.$ $\mathrm{MHz})$ and Galileo E5 $\left(F_{s}=120 \mathrm{MHz}\right)$. SNR out $_{\text {in }}[\mathrm{dB}]$ and coherent integration time $T_{I}$ in [ms].

phase and amplitude estimation using a generic band-limited signals under the narrowband assumption. The latter CRB is particularly useful because it is expressed only from the signal samples. The new CRB was used to characterize the first estimation stage in GNSS delay/phase-based positioning, which leads to new precise positioning receiver design.

\section{REFERENCES}

[1] P. J. Schreier and L. L. Scharf, Statistical Signal Processing of Complex-Valued Data, Cambridge University Press 2010.

[2] F. C. Schweppe, "Sensor array data processing for multiple signal sources", IEEE Trans. on IT, 14: 294-305, 1968

[3] P. Stoica and A. Nehorai, "Performances study of conditional and unconditional direction of arrival estimation," IEEE Trans. on ASSP, 38(10): 1783-1795, 1990.

[4] L. L. Scharf, Statistical signal processing : detection, estimation, and time series analysis, Addison-Wesley, 2002.

[5] H. L. Van Trees, Optimum Array Processing, Wiley-Interscience, 2002.

[6] A. Renaux, P. Forster, E. Chaumette and P. Larzabal, "On the High SNR CML Estimator Full Statistical Characterization", IEEE Trans. Signal Process., 54(12): 4840-4843, 2006.

[7] B. Ottersten, M. Viberg, P. Stoica and A. Nehorai: "Exact and Large Sample Maximum Likelihood Techniques for Parameter Estimation and Detection in Array Processing", Radar Array Processing”, §4, 1993, Springer-Verlag.

[8] J. Tsao, B. D. Steinberg, "Reduction of Sidelobe and Speckle Artifacts in Microwave Imaging: the CLEAN Technique", IEEE Trans. on AP, 36(4): 543-556, 1988.

[9] I. Ziskind and M. Wax, "Maximum likelihood localization of multiple sources by alternating projection", IEEE Trans. on ASSP, 36(10): 1553-1560, 1988.

[10] P. Stoica and T. Söderström, "On reparametrization of loss functions used in estimation and the invariance principle", Signal Processing, vol. 17, pp. 383-387, Aug. 1989.
[11] F. Antreich, J. A. Nossek, G. Seco-Granados and A. L. Swindlehurst, "The extended invariance principle for signal parameter estimation in an unknown spatial field", IEEE Trans. Signal Process. 59 (7):32133225,2011

[12] A. L. Swindlehurst and P. Stoica, "Maximum likelihood methods in radar array signal processing", Proc. IEEE 86 (2):421-441, 1998

[13] F. Vincent, E. Chaumette, C. Charbonnieras, J. Israel, M. Aubault and F. Barbiero, "Asymptotically Efficient GNSS Trilateration", Signal Processing, 137: 270-277, 2017.

[14] P. Closas, C. Fernández-Prades, J. A. Fernández-Rubio, "Maximum likelihood estimation of position in GNSS", IEEE Signal Process. Letters, 14(5):359-362, 2007.

[15] P. J. G. Teunissen, O. Montenbruck (Eds.), Handbook of Global Navigation Satellite Systems, Springer, Switzerland, 2017.

[16] S. Choy, S. Bisnath, C. Rizos, "Uncovering common misconceptions in GNSS Precise Point Positioning and its future prospect", GPS Solutions 2017, 21.

[17] A. Dogandzic and A. Nehorai, "Cramér-Rao bounds for estimating range, velocity, and direction with an active array", IEEE Trans. Signal Process. 49(6): 1122-1137, 2001

[18] N. Noels, H. Wymeersch, H. Steendam and M. Moeneclaey, "True Cramér-Rao bound for timing recovery from a bandlimited linearly modulated waveform with unknown carrier phase and frequency", IEEE Trans. on Commun., 52(3):473-483, 2004.

[19] P. Closas, C. Fernández-Prades, J. A. Fernández-Rubio, "Cramér-Rao bound analysis of positioning approaches in GNSS receivers", IEEE Trans. Signal Process., 57(10):3775-3786, 2009.

[20] C. Enneking et al., "Multi-satellite time-delay estimation for reliable high-resolution GNSS receivers", in: Proc. of the IEEE/ION PLANS, Myrtle Beach, SC, USA, 2012

[21] Y. Chen and R. S. Blum, "On the Impact of Unknown Signals on Delay, Doppler, Amplitude, and Phase Parameter Estimation", IEEE Trans. Signal Process., 67(2):431-443, 2019.

[22] A. Bartov and H. Messer, "Lower bound on the achievable DSP performance for localizing step-like continuous signals in noise", IEEE Trans. Signal Process., 46(8): 2195-2201, 1998.

[23] S.M. Kay, Fundamentals of Statistical Signal Processing: estimation theory, Prentice-Hall, 1993.

[24] S. F. Yau and Y. Bresler, "A Compact Cramér-Rao Bound Expression for Parametric Estimation of Superimposed Signals", IEEE Trans. Signal Process., 40(5): 1226-1230, 1992.

[25] D. C. Rife and R. R. Boorstyn, "Single tone parameter estimation from discrete-time observations", IEEE Trans. on IT, 20(5): 591-598, 1974.

[26] A. N. D'Andrea, U. Mengali and R. Reggiannini, "The modified Cramér-Rao bound and its application to synchronization problems", IEEE Trans. on Commun., 42(2/3/4): 1391-1399, 1994.

[27] S. T. Smith, "Statistical résolution limits and the complexified Cramér Rao bound", IEEE Trans. Signal Process., 53(5): 1597-1609, 2005.

[28] M. N. El Korso, R. Boyer, A. Renaux and S. Marcos, "Conditional and Unconditional Cramér-Rao Bounds for Near-Field Source Localization”, IEEE Trans. Signal Process., 58(5): 2901-2907, 2010.

[29] N. Wu, H. Wang, H. Zhao, H. Liu and J. Kuang, "Evaluation of Cramér-Rao Bounds for Phase Estimation of Coded Linearly Modulated Signals", in Proc. IEEE Vehicular Technology Conference, 2014.

[30] H. Steendam and M. Moeneclaey, "Low-SNR limit of the CramérRao bound for estimating the time-delay of a PSK, QAM, or PAM waveform", IEEE Commun. Letters, 5(1): 31-33, 2001.

[31] H. L. Van Trees, K. L. Bell and Z. Tian, Detection, Estimation and Modulation Theory, Part 1 (2nd Ed), New York, Wiley, 2013.

[32] P. Wang, P. V. Orlik, K. Sadamoto, W. Tsujita and Y. Sawa, "CramérRao Bounds for a Coupled Mixture of Polynomial Phase and Sinusoidal FM Signals", IEEE Signal Process. Letters, 24(6): 746-750, 2017

[33] P. Das, J. Vilà-Valls, E. Chaumette, F. Vincent, L. Davain and S. Bonnabel, "On the accuracy limit of time-delay estimation with a band-limited signal", Proc. of the IEEE Intl. Conf. on Acoustics, Speech and Signal Processing (ICASSP), Brighton, UK, 2019

[34] G. A. F. Seber, Matrix Handbook for Statisticians, Wiley Series in Probability and Statistics, 2008 
APPENDIX I

PROOF OF THE COMPACT CRB EXPRESSION FOR THE Single SOURCE CSM IN SECTION 2

In the following: i) $\left[\begin{array}{ll}\mathbf{A} & \mathbf{B}\end{array}\right]$ and $\left[\begin{array}{l}\mathbf{A} \\ \mathbf{B}\end{array}\right]$ denote the matrix resulting from the horizontal and vertical concatenation of matrices $\mathbf{A}$ and $\mathbf{B}$, ii) $\underline{\mathbf{Y}}=\left[\begin{array}{l}\operatorname{Re}\{\mathbf{Y}\} \\ \operatorname{Im}\{\mathbf{y}\}\end{array}\right], \forall \mathbf{Y} \in \mathbb{C}^{N_{1} \times N_{2}}$, and iii) for the sake of simplicity in the equations $\underline{\mathbf{a}}^{\prime} \triangleq \underline{\mathbf{a}}^{\prime}(\boldsymbol{\theta})$, $\mathbf{a} \triangleq \mathbf{a}(\boldsymbol{\eta})$. Then (2c) can be recast as

$$
\underline{\mathbf{x}}=\underline{\mathbf{a}}^{\prime}(\boldsymbol{\theta}) \rho+\underline{\mathbf{n}}, \underline{\mathbf{n}} \sim \mathcal{N}\left(\mathbf{0}, \sigma_{n}^{2} \mathbf{I}_{2 N} / 2\right), \boldsymbol{\epsilon}^{T}=\left(\sigma_{n}^{2}, \rho, \boldsymbol{\theta}^{T}\right) .
$$

Since $\underline{\mathbf{x}} \sim \mathcal{N}\left(\mathbf{m}_{\underline{\mathbf{x}}}(\boldsymbol{\epsilon}), \mathbf{C}_{\underline{\mathbf{x}}}(\boldsymbol{\epsilon})\right)$, the Fisher information matrix (FIM) is given by the Slepian-Bangs formula [5]

$$
\begin{aligned}
\left(\mathbf{F}_{\boldsymbol{\epsilon}}\right)_{k, l} & =\frac{\partial \mathbf{m}_{\underline{\mathbf{x}}}(\boldsymbol{\epsilon})^{T}}{\partial \epsilon_{k}} \mathbf{C}_{\underline{\mathbf{x}}}^{-1}(\boldsymbol{\epsilon}) \frac{\partial \mathbf{m}_{\underline{\mathbf{x}}}(\boldsymbol{\epsilon})}{\partial \epsilon_{l}} \\
& +\frac{1}{2} \operatorname{tr}\left(\mathbf{C}_{\underline{\mathbf{x}}}^{-1}(\boldsymbol{\epsilon}) \frac{\partial \mathbf{C}_{\underline{\mathbf{x}}}(\boldsymbol{\epsilon})}{\partial \epsilon_{k}} \mathbf{C}_{\underline{\mathbf{x}}}^{-1}(\boldsymbol{\epsilon}) \frac{\partial \mathbf{C}_{\underline{\mathbf{x}}}(\boldsymbol{\epsilon})}{\partial \epsilon_{l}}\right) .
\end{aligned}
$$

In (10), as $\mathbf{m}_{\underline{\mathbf{x}}}(\boldsymbol{\epsilon})=\underline{\mathbf{a}}^{\prime}(\boldsymbol{\theta}) \rho$ and $\mathbf{C}_{\underline{\mathbf{x}}}(\boldsymbol{\epsilon})=\sigma_{n}^{2} / 2 \mathbf{I}_{2 N}$, then

$$
\begin{aligned}
& \mathbf{C R B}_{\boldsymbol{\epsilon}}=\mathbf{F}_{\boldsymbol{\epsilon}}^{-1}=\left[\begin{array}{cc}
\frac{\sigma_{n}^{4}}{N} & \mathbf{0}^{T} \\
\mathbf{0} & \mathbf{C R B}_{(\rho, \boldsymbol{\theta})}
\end{array}\right]
\end{aligned}
$$

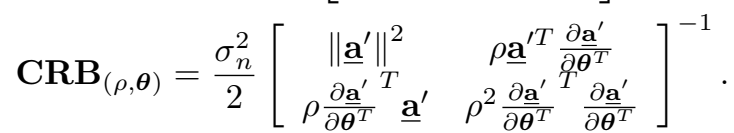

By resorting to the block matrix inversion lemma [34, p 309],

$$
\begin{aligned}
& {\left[\begin{array}{ll}
\mathbf{A}_{11} & \mathbf{A}_{12} \\
\mathbf{A}_{21} & \mathbf{A}_{22}
\end{array}\right]^{-1}=\left[\begin{array}{cc}
\mathbf{C}_{1}^{-1} & -\mathbf{A}_{11}^{-1} \mathbf{A}_{12} \mathbf{C}_{2}^{-1} \\
-\mathbf{C}_{2}^{-1} \mathbf{A}_{21} \mathbf{A}_{11}^{-1} & \mathbf{C}_{2}^{-1}
\end{array}\right],} \\
& \mathbf{C}_{1}=\mathbf{A}_{11}-\mathbf{A}_{12} \mathbf{A}_{22}^{-1} \mathbf{A}_{21}, \mathbf{C}_{2}=\mathbf{A}_{22}-\mathbf{A}_{21} \mathbf{A}_{11}^{-1} \mathbf{A}_{12} \\
& \mathbf{C}_{1}^{-1}=\mathbf{A}_{11}^{-1}+\mathbf{A}_{11}^{-1} \mathbf{A}_{12} \mathbf{C}_{2}^{-1} \mathbf{A}_{21} \mathbf{A}_{11}^{-1},
\end{aligned}
$$

one obtains

$$
\begin{aligned}
C R B_{\rho} & =\frac{\sigma_{n}^{2}}{2}\left(\underline{\mathbf{a}}^{\prime T} \boldsymbol{\Pi}_{\frac{\partial \underline{\mathbf{a}}^{\prime}}{\partial \boldsymbol{\theta}^{T}}}^{\perp} \underline{\mathbf{a}}^{\prime}\right)^{-1}, \\
\mathbf{C R B}_{\boldsymbol{\theta}} & =\frac{\sigma_{n}^{2}}{2 \rho^{2}} \boldsymbol{\Phi}_{\boldsymbol{\theta}}^{-1}, \quad \boldsymbol{\Phi}_{\boldsymbol{\theta}}=\left(\frac{\partial \underline{\mathbf{a}}^{\prime}}{\partial \boldsymbol{\theta}^{T}}\right)^{T} \boldsymbol{\Pi}_{\underline{\mathbf{a}}^{\prime}}^{\perp} \frac{\partial \underline{\mathbf{a}}^{\prime}}{\partial \boldsymbol{\theta}^{T}} .
\end{aligned}
$$

Moreover, since

$$
\begin{aligned}
\underline{\mathbf{A}}^{T} \underline{\mathbf{B}} & =\operatorname{Re}\{\mathbf{A}\}^{T} \operatorname{Re}\{\mathbf{B}\}+\operatorname{Im}\{\mathbf{A}\}^{T} \operatorname{Im}\{\mathbf{B}\} \\
& =\operatorname{Re}\left\{\mathbf{A}^{H} \mathbf{B}\right\}
\end{aligned}
$$

then

$$
\begin{aligned}
& \underline{\mathbf{a}}^{\prime T} \underline{\mathbf{a}}^{\prime}=\mathbf{a}^{H} \mathbf{a}, \\
& \underline{\mathbf{a}}^{\prime T} \frac{\partial \underline{\mathbf{a}}^{\prime}}{\partial \boldsymbol{\theta}^{T}}=\left(\begin{array}{ll}
0 & \left.\operatorname{Re}\left\{\mathbf{a}^{H} \frac{\partial \mathbf{a}}{\partial \boldsymbol{\eta}^{T}}\right\}\right)
\end{array}\right)
\end{aligned}
$$

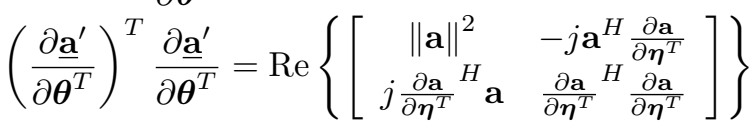

$$
\begin{aligned}
& \left(\frac{\partial \underline{\mathbf{a}}^{\prime}}{\partial \boldsymbol{\theta}^{T}}\right)^{T} \frac{\partial \underline{\mathbf{a}}^{\prime}}{\partial \boldsymbol{\theta}^{T}}=\left[\begin{array}{cc}
\|\mathbf{a}\|^{2} & \operatorname{Im}\left\{\mathbf{a}^{H} \frac{\partial \mathbf{a}}{\partial \boldsymbol{\eta}^{T}}\right\} \\
\operatorname{Im}\left\{\mathbf{a}^{H} \frac{\partial \mathbf{a}}{\partial \boldsymbol{\eta}^{T}}\right\}^{T} & \operatorname{Re}\left\{{\frac{\partial \mathbf{a}}{\partial \boldsymbol{\eta}^{T}}}^{H} \frac{\partial \mathbf{a}}{\partial \boldsymbol{\eta}^{T}}\right\}
\end{array}\right]
\end{aligned}
$$

and therefore

$$
\begin{aligned}
\boldsymbol{\Phi}_{\boldsymbol{\theta}} & =\frac{\partial \underline{\mathbf{a}}^{\prime T}}{\partial \boldsymbol{\theta}^{T}} \frac{\partial \underline{\mathbf{a}}^{\prime}}{\partial \boldsymbol{\theta}^{T}}-\frac{1}{\underline{\mathbf{a}}^{\prime T} \underline{\mathbf{a}}^{\prime}}\left(\underline{\mathbf{a}}^{\prime T} \frac{\partial \underline{\mathbf{a}}^{\prime}}{\partial \boldsymbol{\theta}^{T}}\right)^{T}\left(\underline{\mathbf{a}}^{\prime T} \frac{\partial \underline{\mathbf{a}}^{\prime}}{\partial \boldsymbol{\theta}^{T}}\right) \\
& =\left[\begin{array}{cc}
\|\mathbf{a}\|^{2} & \operatorname{Im}\{\boldsymbol{\Upsilon}\}^{T} \\
\operatorname{Im}\{\boldsymbol{\Upsilon}\} & \operatorname{Re}\{\boldsymbol{\Upsilon}\}-\frac{\operatorname{Re}\{\boldsymbol{\gamma}\} \operatorname{Re}\{\boldsymbol{\gamma}\}^{T}}{\|\mathbf{a}\|^{2}}
\end{array}\right],
\end{aligned}
$$

where $\boldsymbol{\Upsilon}={\frac{\partial \mathbf{a}}{\partial \boldsymbol{\eta}^{T}}}^{H} \frac{\partial \mathbf{a}}{\partial \boldsymbol{\eta}^{T}}$ and $\gamma^{T}=\mathbf{a}^{H} \frac{\partial \mathbf{a}}{\partial \boldsymbol{\eta}^{T}}$, which yields

$$
\begin{aligned}
& \mathbf{\Phi}_{\boldsymbol{\theta}}^{-1}=\left[\begin{array}{cc}
C_{1}^{-1} & -\frac{\mathbf{A}_{12}}{A_{11}} \mathbf{C}_{2}^{-1} \\
-\mathbf{C}_{2}^{-1} \frac{\mathbf{A}_{21}}{A_{11}} & \mathbf{C}_{2}^{-1}
\end{array}\right], \\
& \mathbf{C}_{2}=\operatorname{Re}\{\boldsymbol{\Upsilon}\}-\frac{\operatorname{Re}\{\boldsymbol{\gamma}\} \operatorname{Re}\{\boldsymbol{\gamma}\}^{T}+\operatorname{Im}\{\boldsymbol{\gamma}\} \operatorname{Im}\{\boldsymbol{\gamma}\}^{T}}{\|\mathbf{a}\|^{2}} \\
& =\operatorname{Re}\{\boldsymbol{\Upsilon}\}-\frac{\operatorname{Re}\left\{\boldsymbol{\gamma} \boldsymbol{\gamma}^{H}\right\}}{\|\mathbf{a}\|^{2}}=\operatorname{Re}\left\{\frac{\partial \mathbf{a}}{\partial \boldsymbol{\eta}^{T}} \mathbf{\Pi}_{\mathbf{a}}^{\perp} \frac{\partial \mathbf{a}}{\partial \boldsymbol{\eta}^{T}}\right\} \\
& C_{1}^{-1}=\frac{1}{\|\mathbf{a}\|^{2}}+\frac{\operatorname{Im}\{\boldsymbol{\gamma}\}^{T} \mathbf{C}_{2}^{-1} \operatorname{Im}\{\boldsymbol{\gamma}\}}{\|\mathbf{a}\|^{4}} \\
& \mathbf{C}_{2}^{-1} \frac{\mathbf{A}_{21}}{A_{11}}=\frac{\mathbf{C}_{2}^{-1} \operatorname{Im}\{\boldsymbol{\gamma}\}}{\|\mathbf{a}\|^{2}},
\end{aligned}
$$

that is, the expressions in (3a)-(3d). Last, to obtain (3e) consider that

$$
\begin{aligned}
& \underline{\mathbf{a}}^{\prime T} \boldsymbol{\Pi}_{\frac{\partial \underline{\mathbf{a}}^{\prime}}{\partial \boldsymbol{\theta}^{T}}}^{\perp} \underline{\mathbf{a}}^{\prime}=\mathbf{a}^{H} \mathbf{a}-\left(\begin{array}{c}
0 \\
\operatorname{Re}\{\gamma\}
\end{array}\right)^{T} \\
& \times\left[\begin{array}{cc}
\mathbf{a}^{H} \mathbf{a} & \operatorname{Im}\{\boldsymbol{\gamma}\}^{T} \\
\operatorname{Im}\{\boldsymbol{\gamma}\} & \operatorname{Re}\{\boldsymbol{\Upsilon}\}
\end{array}\right]^{-1}\left(\begin{array}{c}
0 \\
\operatorname{Re}\{\boldsymbol{\gamma}\}
\end{array}\right) \\
& =\mathbf{a}^{H} \mathbf{a}-\operatorname{Re}\{\boldsymbol{\gamma}\}^{T}\left(\operatorname{Re}\{\boldsymbol{\Upsilon}\}-\frac{\operatorname{Im}\{\boldsymbol{\gamma}\} \operatorname{Im}\{\boldsymbol{\gamma}\}^{T}}{\mathbf{a}^{H} \mathbf{a}}\right)^{-1} \operatorname{Re}\{\boldsymbol{\gamma}\} \\
& =\mathbf{a}^{H} \mathbf{a}-\operatorname{Re}\{\boldsymbol{\gamma}\}^{T}\left(\mathbf{C}_{2}+\frac{\operatorname{Re}\{\gamma\} \operatorname{Re}\{\gamma\}^{T}}{\mathbf{a}^{H} \mathbf{a}}\right)^{-1} \operatorname{Re}\{\gamma\} \\
& =\mathbf{a}^{H} \mathbf{a}-\operatorname{Re}\{\boldsymbol{\gamma}\}^{T} \frac{\mathbf{a}^{H} \mathbf{a C}_{2}^{-1} \operatorname{Re}\{\boldsymbol{\gamma}\}}{\mathbf{a}^{H} \mathbf{a}+\operatorname{Re}\{\boldsymbol{\gamma}\}^{T} \mathbf{C}_{2}^{-1} \operatorname{Re}\{\boldsymbol{\gamma}\}} \\
& \underline{\mathbf{a}}^{\prime T} \boldsymbol{\Pi}_{\frac{\partial \mathbf{a}^{\prime}}{\partial \boldsymbol{\theta}^{T}}}^{\perp} \underline{\mathbf{a}}^{\prime}=\frac{\left(\mathbf{a}^{H} \mathbf{a}\right)^{2}}{\mathbf{a}^{H} \mathbf{a}+\operatorname{Re}\{\gamma\}^{T} \mathbf{C}_{2}^{-1} \operatorname{Re}\{\gamma\}}, \\
& C R B_{\rho}=\frac{\sigma_{n}^{2}}{2 \mathbf{a}^{H} \mathbf{a}}\left(1+\frac{\operatorname{Re}\{\gamma\}^{T} \mathbf{C}_{2}^{-1} \operatorname{Re}\{\gamma\}}{\mathbf{a}^{H} \mathbf{a}}\right) .
\end{aligned}
$$

APPENDIX II

PROOF OF THE CRB EXPRESSION FOR THE NARrowband Signal Model in SECTION 3

First notice that

$\mathbf{C R B}_{\boldsymbol{\eta}}=\frac{\sigma_{n}^{2}}{2 \rho^{2}} \boldsymbol{\Phi}_{\boldsymbol{\eta}}^{-1}$

$\boldsymbol{\Phi}_{\boldsymbol{\eta}}=\lim _{\left(N_{1}^{\prime}, N_{2}^{\prime}\right) \rightarrow(-\infty,+\infty)} \operatorname{Re}\left\{\left(\frac{\partial \mathbf{a}(\boldsymbol{\eta})}{\partial \boldsymbol{\eta}^{T}}\right)^{H} \boldsymbol{\Pi}_{\mathbf{a}(\boldsymbol{\eta})}^{\perp} \frac{\partial \mathbf{a}(\boldsymbol{\eta})}{\partial \boldsymbol{\eta}^{T}}\right\}$. 
The derivative of $a(t ; \boldsymbol{\eta})$ w.r.t. the parameters of interest reads

$$
\begin{aligned}
& \frac{\partial a(t ; \boldsymbol{\eta})}{\partial \boldsymbol{\eta}}=-\mathbf{Q} \boldsymbol{\vartheta}(t-\tau) e^{-j \omega_{c} b(t-\tau)} \\
& \mathbf{Q}=\left[\begin{array}{ccc}
-j \omega_{c} b & 0 & 1 \\
0 & j \omega_{c} & 0
\end{array}\right], \boldsymbol{\vartheta}(t)=\left(\begin{array}{c}
c(t) \\
t c(t) \\
c^{(1)}(t)
\end{array}\right) .
\end{aligned}
$$

where $c^{(1)}(t)=\frac{d c(t)}{d t}$. Then we can write

$$
\begin{aligned}
& \mathbf{a}^{H}(\boldsymbol{\eta}) \frac{\partial \mathbf{a}(\boldsymbol{\eta})}{\partial \boldsymbol{\eta}^{T}} \\
& =-\left(\mathbf{Q} \sum_{n=N_{1}^{\prime}}^{N_{2}^{\prime}} \boldsymbol{\vartheta}\left(n T_{s}-\tau\right) c\left(n T_{s}-\tau\right)^{*}\right)^{T}, \\
& \frac{\partial \mathbf{a}(\boldsymbol{\eta})^{H}}{\partial \boldsymbol{\eta}^{T}} \frac{\partial \mathbf{a}(\boldsymbol{\eta})}{\partial \boldsymbol{\eta}^{T}} \\
& =\mathbf{Q}^{*}\left(\sum_{n=N_{1}^{\prime}}^{N_{2}^{\prime}} \boldsymbol{\vartheta}\left(n T_{s}-\tau\right) \boldsymbol{\vartheta}^{H}\left(n T_{s}-\tau\right)\right) \mathbf{Q}^{T}, \\
& \|\mathbf{a}(\boldsymbol{\eta})\|^{2}=\sum_{n=N_{1}^{\prime}}^{N_{2}^{\prime}}\left|c\left(n T_{s}-\tau\right)\right|^{2},
\end{aligned}
$$

and

$$
\begin{aligned}
& \lim _{\left(N_{1}^{\prime}, N_{2}^{\prime}\right) \rightarrow(-\infty,+\infty)} T_{s} \sum_{n=N_{1}^{\prime}}^{N_{2}^{\prime}} \boldsymbol{\vartheta}\left(n T_{s}-\tau\right) c\left(n T_{s}-\tau\right)^{*} \\
& =\int_{-\infty}^{+\infty} \boldsymbol{\vartheta}(t-\tau) c(t-\tau)^{*} d t=\int_{-\infty}^{+\infty} \boldsymbol{\vartheta}(t) c(t)^{*} d t=\mathbf{w} \\
& \lim _{\left(N_{1}^{\prime}, N_{2}^{\prime}\right) \rightarrow(-\infty,+\infty)} T_{s} \sum_{n=N_{1}^{\prime}}^{N_{2}^{\prime}} \boldsymbol{\vartheta}\left(n T_{s}-\tau\right) \boldsymbol{\vartheta}^{H}\left(n T_{s}-\tau\right) \\
& =\int_{-\infty}^{+\infty} \boldsymbol{\vartheta}(t-\tau) \boldsymbol{\vartheta}(t-\tau)^{H} d t=\int_{-\infty}^{+\infty} \boldsymbol{\vartheta}(t) \boldsymbol{\vartheta}(t)^{H} d t=\mathbf{W}
\end{aligned}
$$

with

$$
\mathbf{w}=\left(\begin{array}{l}
w_{1} \\
w_{2} \\
w_{3}
\end{array}\right), \quad \mathbf{W}=\left[\begin{array}{ccc}
w_{1} & w_{2}^{*} & w_{3}^{*} \\
w_{2} & W_{2,2} & \varpi^{*} \\
w_{3} & \varpi & W_{3,3}
\end{array}\right],
$$

where $\varpi=\int_{-\infty}^{+\infty} t c^{(1)}(t) c(t)^{*} d t$, and $w_{1}, w_{2}, W_{2,2}, W_{3,3} \in$ $\mathbb{R}$. From these results, we can write that $\boldsymbol{\Phi}_{\boldsymbol{\eta}}$ is

$$
\begin{aligned}
\boldsymbol{\Phi}_{\boldsymbol{\eta}} & =F_{s} \operatorname{Re}\left\{\mathbf{Q} \mathbf{W} \mathbf{Q}^{H}-\frac{(\mathbf{Q w})(\mathbf{Q w})^{H}}{w_{1}}\right\} \\
& =F_{s}\left[\begin{array}{cc}
W_{3,3}-\frac{\left|w_{3}\right|^{2}}{w_{1}} & \omega_{c} \operatorname{Im}\left\{\varpi-\frac{w_{2} w_{3}}{w_{1}}\right\} \\
\omega_{c} \operatorname{Im}\left\{\varpi-\frac{w_{2} w_{3}}{w_{1}}\right\} & \omega_{c}^{2}\left(W_{2,2}-\frac{w_{2}^{2}}{w_{1}}\right)
\end{array}\right],
\end{aligned}
$$

where from [33] we already have $w_{1}, w_{3}$ and $W_{3,3}$,

$$
\begin{aligned}
& w_{1}=\frac{\mathbf{c}^{H} \mathbf{c}}{F_{s}}, \\
& w_{3}=\mathbf{c}^{H} \boldsymbol{\Lambda} \mathbf{c}, \\
& W_{3,3}=F_{s} \mathbf{c}^{H} \mathbf{V} \mathbf{c},
\end{aligned}
$$

and the remaining terms are computed as

$$
\begin{aligned}
& w_{2}=\int_{-\infty}^{+\infty} c(t)(t c(t))^{*} d t \\
& =\int_{-\frac{F_{s}}{2}}^{\frac{F_{s}}{2}} c(f)\left(\frac{j}{2 \pi} \frac{d c(f)}{d f}\right)^{*} d f \\
& =\frac{1}{F_{s}^{2}} \int_{-\frac{1}{2}}^{\frac{1}{2}}\left((\mathbf{D c})^{H} \boldsymbol{v}(f)\right)\left(\boldsymbol{v}^{H}(f) \mathbf{c}\right) d f \\
& =\frac{1}{F_{s}^{2}} \mathbf{c}^{H} \mathbf{D}^{H}\left(\int_{-\frac{1}{2}}^{\frac{1}{2}} \boldsymbol{v}(f) \boldsymbol{v}^{H}(f) d f\right) \mathbf{c} \\
& =\frac{1}{F_{s}^{2}} \mathbf{c}^{H} \mathbf{D} \mathbf{c} \text {, } \\
& \varpi=\int_{-\infty}^{+\infty} c^{(1)}(t)(t c(t))^{*} d t \\
& =\int_{-\frac{F_{s}}{2}}^{\frac{F_{s}}{2}}(j 2 \pi f) c(f)\left(\frac{j}{2 \pi} \frac{d c(f)}{d f}\right)^{*} d f \\
& =\frac{1}{F_{s}} \int_{-\frac{1}{2}}^{\frac{1}{2}}(j 2 \pi f)\left(\boldsymbol{v}^{H}(f) \mathbf{c}\right)\left((\mathbf{D c})^{H} \boldsymbol{v}(f)\right) d f \\
& =\frac{1}{F_{s}} \mathbf{c}^{H} \mathbf{D}^{H}\left(j 2 \pi \int_{-\frac{1}{2}}^{\frac{1}{2}} f \boldsymbol{v}(f) \boldsymbol{v}^{H}(f) d f\right) \mathbf{c} \\
& =\frac{1}{F_{s}} \mathbf{c}^{H} \mathbf{D} \boldsymbol{\Lambda} \mathbf{c}, \\
& W_{2,2}=\int_{-\infty}^{+\infty}|t c(t)|^{2} d t=\int_{-\frac{F_{s}}{2}}^{\frac{F_{s}}{2}}\left|\frac{j}{2 \pi} \frac{d c(f)}{d f}\right|^{2} d f \\
& =\frac{1}{F_{s}^{3}} \int_{-\frac{1}{2}}^{\frac{1}{2}}\left|\boldsymbol{v}^{H}(f)(\mathbf{D c})\right|^{2} d f \\
& =\frac{1}{F_{s}^{3}} \mathbf{c}^{H} \mathbf{D}^{H}\left(\int_{-\frac{1}{2}}^{\frac{1}{2}} \boldsymbol{v}(f) \boldsymbol{v}^{H}(f) d f\right) \mathbf{D} \mathbf{c} \\
& =\frac{1}{F_{s}^{3}} \mathbf{c}^{H} \mathbf{D}^{2} \mathbf{c} \text {. }
\end{aligned}
$$

Finally, the other terms in (8b)-(8d) are also computed from $\mathbf{w}$ as follows:

$$
\begin{aligned}
& C R B_{\varphi}=\frac{\sigma_{n}^{2}}{2 \rho^{2}} \frac{1}{F_{s} w_{1}}+\frac{1}{w_{1}^{2}} \times \\
& \left(\begin{array}{l}
\operatorname{Im}\left\{w_{3}\right\}-b \omega_{c} w_{1} \\
\omega_{c} w_{2}
\end{array}\right)^{T} \mathbf{C R B}_{\boldsymbol{\eta}}\left(\begin{array}{l}
\operatorname{Im}\left\{w_{3}\right\}-b \omega_{c} w_{1} \\
\omega_{c} w_{2}
\end{array}\right), \\
& \mathbf{C R B}_{\boldsymbol{\eta}, \varphi}=\mathbf{C R B}_{\boldsymbol{\eta}} \frac{1}{w_{1}}\left(\begin{array}{l}
\operatorname{Im}\left\{w_{3}\right\}-b \omega_{c} w_{1} \\
\omega_{c} w_{2}
\end{array}\right), \\
& C R B_{\rho}=\frac{\sigma_{n}^{2}}{2 F_{s} w_{1}}+\rho^{2} \frac{1}{w_{1}^{2}} \times \\
& \left(\begin{array}{l}
\operatorname{Re}\left\{w_{3}\right\} \\
0
\end{array}\right)^{T} \mathbf{C R B}_{\boldsymbol{\eta}}\left(\begin{array}{l}
\operatorname{Re}\left\{w_{3}\right\} \\
0
\end{array}\right)^{T} .
\end{aligned}
$$

